\title{
From identification of fluorescent flavoproteins to mitochondrial redox indicators in intact tissues
}

\author{
Ilmo E. Hassinen \\ Department of Medical Biochemistry and Molecular Biology \\ Institute of Biomedicine \\ University of Oulu, P.O. Box 5000, FIN-90014, Oulu, Finland \\ ilmo.hassinen@oulu.fi
}

Received 30 July 2013

Accepted 17 September 2013

Published 18 November 2013

\begin{abstract}
Development of the use of flavin and nicotinamide-adenine nucleotide fluorescence in monitoring the redox state of the free mitochondrial $\mathrm{NADH} / \mathrm{NAD}^{+}$couple in cells, tissues and organs is reviewed. A break-through was the identification of dihydrolipoamide dehydrogenase $(\mathrm{FpL})$ as the major NAD-linked fluorescent flavoprotein of mitochondria. This mitochondrial matrix flavoprotein is in equilibrium with the free $\mathrm{NADH} / \mathrm{NAD}^{+}$pool and its mid-potential is sufficiently near to that of $\mathrm{NADH} / \mathrm{NAD}^{+}$so that its percentage reduction follows that of the latter. Possibilities of monitoring mitochondrial and cytosolic NADH depend on the population density of mitochondria and thus are tissue-dependent. Upon a shift toward reduction, fluorescence intensities of $\mathrm{NADH}$ and flavins swing to reciprocal directions, so that the NADH/flavin fluorescence ratio can be used to increase the sensitivity of redox monitoring. This method is attaining widening use in studies on metabolic regulation under normal and pathological conditions.
\end{abstract}

Keywords: Flavin-adenine dinucleotide; dihydrolipoamide dehydrogenase; electron transfer flavoprotein; flavin mononucleotide; compartment-specific monitoring.

\section{Development of Views on Flavopro- teins of Respiratory Chain Complex I in $1967-1968$}

The common knowledge in the early sixties was that amongst the enzyme complexes of the respiratory chain Complex I (NADH:ubiquinone oxidoreductase) and Complex II (succinate:ubiquinone oxidoreductase) were flavoproteins. The former contained flavin mononucleotide (FMN) and the latter flavin-adenine dinucleotide (FAD). In 1967, Chance and coworkers observed that antimycin A in the presence of an $\mathrm{NAD}^{+}$-linked oxidizable substrate in suspension of submitochondrial particles caused a pronounced flavin fluorescence decrease concomitantly with an absorbance decrease at

This is an Open Access article published by World Scientific Publishing Company. It is distributed under the terms of the Creative Commons Attribution 3.0 (CC-BY) License. Further distribution of this work is permitted, provided the original work is properly cited. 
wavelengths attributable to flavins. Subsequent addition of rotenone caused a partial reoxidation. Similar experiments on intact mitochondria led to the conclusion that Complex I contained two flavins, one (FpD1) of the NADH side and the other (FpD2) on the oxygen side of the rotenone-binding site. $^{1,2}$ Further studies on rat liver mitochondria and submitochondrial particles at the Johnson Foundation, however, revealed, that the high-fluorescence, low-potential flavin $(\mathrm{FpF} 1)$ resides in the soluble mitochondrial matrix, whereas the low-fluorescence, NADH-linked flavin with higher redox potential was membrane bound. Reoxidation of the high-fluorescence, low-potential flavin was sensitive to arsenite, which binds to vicinal dithiols, and its redox mid-potential was compatible with the dihydrolipoamide dehydrogenase $(\mathrm{FpL})$. So it was most likely, that the high-fluorescence, low-potential flavin was a component of the 2-oxoacid dehydrogenase complexes, not of the respiratory chain. ${ }^{3}$ Titrations with the $\beta$-hydroxybutyrate/acetoacetate pair gave a mid-potential of $-305 \mathrm{mV}$ for the main fluorescent flavin component. These data were later corroborated by means of anaerobic potentiometric titration employing a platinum electrode and redox mediator dyes. ${ }^{4}$ Thus, the mid-potential of $\mathrm{FpL}$ is not far from the generally accepted $-320-\mathrm{mV} E_{m}$ of the $\mathrm{NADH} / \mathrm{NAD}^{+}$couple. The rapidity of the fluorescence response to the $\mathrm{NADH} / \mathrm{NAD}^{+}$ratio poised by the $\beta$-hydroxybutyrate dehydrogenase indicates that there is a near equilibrium between $\mathrm{FpL}$ and $\mathrm{NADH} / \mathrm{NAD}^{+}$that makes $\mathrm{FpL}$ fluorescence to a sensitive compartment-specific mitochondrial redox indicator particularly in heart muscle, where the mitochondrial-free $\mathrm{NADH} / \mathrm{NAD}^{+}$pair in an isolated, perfused beating rat heart stays at $-314 \mathrm{mV}$ under basal conditions as estimated from the glutamate dehydrogenase equilibrium. ${ }^{5}$

After these refinements of the identities of mitochondrial flavins by means of spectrophotometric, fluorometric and redox potential measurements it became obvious that Complex I contained only one flavin moiety, FMN in a low-fluorescence state. ${ }^{6}$

\section{Fluorescent Flavin Becomes a Compartment-Specific Mitochondrial Redox Probe}

Due to its extreme complexity and large size of Complex I, revelation of its structural details and mechanism of function had to await introduction of the era of molecular biology and progress in methodology of membrane enzyme purification and crystallography. The complete crystal structure of the functional core of respiratory Complex I including its redox centers has been recently resolved at $3.3-\AA$ resolution. ${ }^{7}$ After disproval of its role as an electron carrier of Complex I, the flavin previously named FpD1 was given another role in serving with its new identity (FpL) as a mitochondrial matrixspecific probe for redox state of the $\mathrm{NADH} / \mathrm{NAD}^{+}$ couple. Chance and coworkers in Philadelphia ${ }^{8-11}$ and our group in Helsinki ${ }^{12}$ and Oulu ${ }^{5,13-15}$ began soon exploiting flavin fluorescence as a mitochondrial redox probe for cells, tissues and organs.

For the redox state of NADH, the need of a compartment-specific indicator was clearly shown by monitoring $\mathrm{NADH}+\mathrm{NADPH}$ fluorescence by surface fluorescence in isolated perfused rat hearts and livers during variation of the cytoplasmic $\mathrm{NADH} / \mathrm{NAD}^{+}$ratio to by means of infusing lactate and pyruvate in varying ratios. ${ }^{15}$ In the liver, $\mathrm{NADH}$ fluorescence increased upon increase in the [lactate]/[pyruvate] ratio, but in the heart the response was opposite, as NADH fluorescence increased upon decrease in the [lactate]/[pyruvate] ratio. In both cases, the [lactate]/[pyruvate] ratio was adjusted by changing the infusion rate of pyruvate. This behavior is evidently caused by pyruvate oxidation in mitochondria which lack lactate dehydrogenase. The finding indicates that the total NADH fluorescence changes in the liver under certain conditions that reflect the cytosolic NADH/ $\mathrm{NAD}^{+}$ratio, whereas in the heart the majority of NADH fluorescence originates from mitochondria in a situation where the mitochondrial and cytosolic redox states swing to opposite direction. Moreover, this analysis shows that cytosolic $\mathrm{NADH} / \mathrm{NAD}^{+}$ ratio cannot be fluorometrically monitored in the heart, ${ }^{15}$ so that one must resort to tissue sampling and determination of concentrations of oxidized and reduced components of suitable indicator redox substrate pair. The fact, that cytosol does not significantly contribute to the NADH fluorescence from intact myocardium, can be used to simplify redox data processing, as exemplified in a thermodynamic study of respiratory control in heart muscle. ${ }^{16}$ Indeed, early observations had already suggested that metabolically labile NADH fluorescence of muscle tissue comes mainly from mitochondria. ${ }^{17}$ 
It must be born in mind that FpL is not the only fluorescent mitochondrial flavoprotein. Kunz and co-workers have with differential fluorescence spectrometry shown that although a dominant part of the flavin fluorescence comes from the FpL, also other flavoproteins are detectable under defined conditions. ${ }^{18-20}$ They calculated that about $50 \%$ of the flavin fluorescence was NAD linked and originated from FpL displaying an $E_{m}$ value of $-283 \mathrm{mV}$ for the flavin. About $25 \%$ of the flavin fluorescence was not $\mathrm{NAD}^{+}$linked, and this was tentatively identified as the electron transfer flavoprotein (ETF) of the $\beta$-oxidation system showing an $E_{m}$ value of $-52 \mathrm{mV}$. These values generally corroborate earlier observations. ${ }^{4}$ According to the substrate screening performed by Kunz and Kunz, ${ }^{18}$ additions of glycerol-3-phosphate, proline and choline to rat liver mitochondria did not result in noticeable decrease in the fluorescence of non-NADlinked flavoproteins, which indicates that the flavoenzymes glycerol-3-phosphate dehydrogenase (glycerol-3-phosphate:ubiquinone oxidoreductase), proline dehydrogenase and choline oxidase cannot be monitored by fluorescence. The experiments by Ragan and Garland ${ }^{21}$ corroborate this by showing that, although the glycerol phosphate oxidase activity of submitochondrial particles from Torulopsis utilis is high, its substrate only causes a paradoxical flavin fluorescence increase, although the concomitant absorbance decrease in the "flavin through" region denotes flavin reduction. A plausible explanation is that the fluorescence increase was due to a decrease in the internal filter effect at the flavin excitation band, revealing the fluorescence of other (oxidized) flavoproteins. ${ }^{21}$ The rest of flavoproteins are reducible with dithionite only. ${ }^{18-21}$ An additive contribution of FpL and ETF on flavin fluorescence is probably the cause of the extreme flavin fluorescence decrease (by taking the anoxia response as a reference) upon infusion on medium chain fatty acid into an isolated perfused rat heart, because $\beta$-oxidation will feed electrons both via the $\mathrm{NAD}^{+}$ pool and ETF. ${ }^{22,23}$

Free riboflavin, FMN and FAD have three main absorbance bands centered on 268, 374 and $449 \mathrm{~nm}$, and the fluorescence excitation spectrum is identical to the absorbance spectrum. The widths of the absorption bands allow finding of proper lasers for fluorescence excitation. The emission band is broad with a maximum at $530 \mathrm{~nm} .{ }^{24}$ The fluorescence yields of riboflavin and FMN are almost equal, but the fluorescence intensity of FAD is only $15 \%$ of that of riboflavin. ${ }^{24}$ It must, however, be emphasized that the fluorescence of bound flavin prosthetic group in an enzyme is strongly dependent on the apoenzyme. In most cases, FAD fluorescence is quenched upon incorporation into a peptide, but this is not the case for lipoamide dehydrogenase. ${ }^{25}$ For example, FMN in Complex $\mathrm{I}$ is practically nonfluorescent, whereas lipoamide dehydrogenase is one of the enzymes, which increase FAD fluorescence upon biding. The emission peak of lipoamide dehydrogenase is red-shifted by $27 \mathrm{~nm}$ in comparison with ETF ${ }^{20}$ and on this basis ETF fluorescence has been used to trace cellular fatty acid oxidation. ${ }^{26}$ However, fluorescence quenching of ETF plus fatty acyl-CoA dehydrogenase upon reduction obtained by a fatty acyl carnitine ester in liver mitochondria is only $5 \%$ of that of dihydrolipoyl dehydrogenase upon addition of an $\mathrm{NAD}^{+}$-linked substrate, when expressed as $\Delta$-fluorescence $/ \Delta$-absorbance ratio. ${ }^{2}$ This may degrade the specificity of fluorescence monitoring of ETF in intact tissues.

\section{Monitoring Fluorescence of Flavin or NADH + NADPH or Both?}

Flavoprotein fluorescence intensity in different tissues and organs is variable and depends on mitochondrial population density and metabolic pattern. Moreover, converting the fluorescence reading to absolute redox potential values is not trivial. In many cases, the fully oxidized and fully reduced conditions are used as calibration reference points, but as emphasized by Bücher and coworkers ${ }^{27}$ uncertainty remains whether these conditions can be reached in living cells. They introduced a method of double reciprocal plotting of changes in fluorescence and indicator metabolite ratio to extrapolate the fully oxidized and fully reduced states. ${ }^{27}$

The calibration of flavoprotein fluorescence is also hampered by background caused by the fluorescence of lipofuscin, a mixture of debris contained in lysosomes. The composition of lipofuscin has not been resolved completely and appears to be dependent on the tissue. In the retina, it has been shown to be composed mainly of bisretinoids, ${ }^{28}$ but in the liver it contains redox components originating from lysosomal membranes, which contain even FAD and ubiquinone. ${ }^{29}$ The lysosomes have been demonstrated 
also to contain FAD phosphohydrolase, ${ }^{30}$ which may allow lysosomal accumulation of more fluorescent flavin compounds. It remains to be established, whether this fluorescent debris is metabolically reactive. In any case, lipofuscin produces a fluorescence background, which is challenging to remove without organelle-specific scanning and which influences the redox calibration of flavoprotein fluorescence in tissues containing lipofuscin.

Upon mitochondrial oxidation and reduction, the fluorescence intensities of flavin and NADH swing to opposite directions, so that the $\mathrm{NADH} /$ flavin fluorescence ratio represents an amplified signal of $\mathrm{NADH} / \mathrm{NAD}^{+}$increase. However, this presumes that the detectable flavin and NADH signals come from the same intracellular compartment, which actually holds in the case of heart muscle. ${ }^{15}$ The cytosolic-free NADH concentration calculated from the lactate dehydrogenase equilibrium constant has been considered too low to be detected by surface fluorometry of a perfused liver, and it has been concluded that the [lactate]/[pyruvate] ratio responsive NADH fluorescence originates from cytosolic protein-bound nucleotide pool, because binding generally enhances NADH fluorescence. ${ }^{27}$ This being the case, in liver the contribution of cytosol to total NADH fluorescence is stronger than in the heart muscle, where fluorescent flavoprotein and NADH report mitochondrial redox state as noted above. ${ }^{15,17}$

The low-temperature enhancement of fluorescence quantum yield was first employed in mitochondrial research by Chance and coworkers in 1979 and applied to the determination of NADH/ flavin fluorescence ratios in freeze-trapped mitochondrial suspensions. ${ }^{31}$ Since then, redox mapping of freeze-trapped tissues in two and three dimensions have been successfully applied to liver by exposing consecutive layers of frozen tissue for scanning. ${ }^{32,33}$ The NADH/flavin fluorescence has gradually obtained more general use in biomedical research, and "normalized optical redox ratio" modifications such as Flavin/(Flavin + NADH) or $\mathrm{NADH} /($ Flavin $+\mathrm{NADH})$ have been proposed to be less affected by hemodynamic changes or mitochondrial population density. ${ }^{11,34}$ These modified ratios produce data which better obey the normal distribution $^{35}$ and also compress them to a zero-toone range. Also other means of normalizing the flavin/NADH fluorescence ratio scans are in use. $^{36,37}$
The inventions of confocal ${ }^{38}$ laser scanning fluorescence microscopy, and two-photon excitation fluorometry ${ }^{39}$ or their combination have widened the application range of redox scanning by means of autofluorescence of flavoproteins and reduced nicotinamide nucleotides in cells and tissues. This is particularly true with monitoring of $\mathrm{NAD}(\mathrm{P}) \mathrm{H}$ fluorescence, because one-photon excitation necessitates a near-UV light source, whereas two-photon excitation can be accomplished with a near-IR wavelength, which penetrates deeper in tissue. This method also limits potential photodecomposition to the focal point so that surrounding cells are less affected. In contrast to confocal microscopy, no pinholes are necessary in two-photon excitation microscopy to remove the background. For flavin/NAD (P)H work, two-photon excitation is often applied for $\mathrm{NAD}(\mathrm{P}) \mathrm{H}$ and one-photon excitation of flavoproteins, although two-photon excitation is applicable also to the flavins. These methods have been applied for investigating mitochondrial subpopulations in cardiomyocytes, ${ }^{40}$ mitochondrial regulation and organization in cells and skinned muscle fibers influenced by cytoskeletal proteins ${ }^{41,42}$ and research on mitochondrial neuromuscular disorders. ${ }^{43}$

\section{Conclusions}

The Complex I flavin content study, which was initiated in Johnson Foundation, Department of Biochemistry and Biophysics in 1966-1967, led first to an unorthodox interpretation of data, but further investigations ended up in surprising findings, which guided the way to utilization of the dihydrolipoamide dehydrogenase flavin as a practical probe for the redox state of the free $\mathrm{NADH} / \mathrm{NAD}^{+}$ couple in mitochondrial matrix space. This allows nondestructive metabolic characterization of intact tissues both in normal and pathological conditions and is currently obtaining widening use also in clinical applications, particularly by using the $\mathrm{NADH} /$ flavin fluorescence ratio which gives an amplified NADH-dependent redox signal compared with the NADH or flavin fluorescence alone.

\section{References}

1. B. Chance, L. Ernster, P. B. Garland, C. P. Lee, P. A. Light, T. Ohnishi, C. I. Ragan, D. Wong, "Flavoproteins of the mitochondrial respiratory 
chain," Proc. Natl. Acad. Sci. USA 57, 1498-1505 (1967).

2. P. B. Garland, B. Chance, L. Ernster, C. P. Lee, D. Wong, "Flavoproteins of mitochondrial fatty acid oxidation," Proc. Natl. Acad. Sci. USA 58, 16961702 (1967).

3. I. Hassinen, B. Chance, "Oxidation-reduction properties of the mitochondrial flavoprotein chain," Biochem. Biophys. Res. Commun. 31, 895-900 (1968).

4. H. Voltti, I. E. Hassinen, "Oxidation-reduction midpoint potentials of mitochondrial flavoproteins and their intramitochondrial localization," J. Bioenerg. Biomembr. 10, 45-58 (1978).

5. I. E. Hassinen, K. Hiltunen, "Respiratory control in isolated perfused rat heart. Role of the equilibrium relations between the mitochondrial electron carriers and the adenylate system," Biochim. Biophys. Acta 408, 319-330 (1975).

6. C. I. Ragan, P. B. Garland, "The intra-mitochondrial localization of flavoproteins previously assigned to the respiratory chain," Eur. J. Biochem. 10, 399-410 (1969).

7. R. Baradaran, J. M. Berrisford, G. S. Minhas, L. A. Sazanov, "Crystal structure of the entire respiratory complex I," Nature 494, 443-448 (2013).

8. R. Scholz, R. G. Thurman, J. R. Williamson, B. Chance, T. Bücher, "Flavin and pyridine nucleotide oxidation-reduction changes in perfused rat liver. I. Anoxia and subcellular localization of fluorescent flavoproteins," J. Biol. Chem. 244, 2317-2324 (1969).

9. H. Franke, C. H. Barlow, B. Chance, "Fluorescence of pyridine nucleotide and flavoproteins as an indicator of substrate oxidation and oxygen demand of the isolated perfused rat kidney," Int. J. Biochem. 12, 269-275 (1980).

10. A. Mayevsky, H. Kaplan, J. Haveri, J. Haselgrove, B. Chance, "Three-dimensional metabolic mapping of the freeze-trapped brain: Effects of ischemia in the mongolian gerbil," Brain Res. 367, 63-72 (1986).

11. H. N. Xu, S. Nioka, B. Chance, L. Z. Li, "Heterogeneity of mitochondrial redox state in premalignant pancreas in a PTEN null transgenic mouse model," Adv. Exp. Med. Biol. 701, 207-213 (2011).

12. I. Hassinen, R. H. Ylikahri, "Absorption spectrophotometry of perfused rat liver applied to fructoseinduced inhibition of respiration," Biochem. Biophys. Res. Commun. 38, 1091-1097 (1970).

13. K. Kiviluoma, I. Hassinen, "Role of acetaldehyde and acetate in the development of ethanol-induced cardiac lipidosis, studied in isolated perfused rat hearts," Alcohol Clin. Exp. Res. 7, 169-175 (1983).

14. I. E. Hassinen, "Reflectance spectrophotometric and surface fluorometric methods for measuring the redox state of nicotinamide nucleotides and flavins in intact tissues," Methods Enzymol. 123, 311-320 (1986).

15. E. M. Nuutinen, "Subcellular origin of the surface fluorescence of reduced nicotinamide nucleotides in the isolated perfused rat heart," Basic Res. Cardiol. 79, 49-58 (1984).

16. I. Hassinen, K. Ito, S. Nioka, B. Chance, "Mechanism of fatty acid effect on myocardial oxygen consumption. A phosphorus NMR study," Biochim. Biophys. Acta 1019, 73-80 (1990).

17. J. B. Chapman, "Fluorometric studies of oxidative metabolism in isolated papillary muscle of the rabbit," J. Gen. Physiol. 59, 135-154 (1972).

18. W. S. Kunz, W. Kunz, "Contribution of different enzymes to flavoprotein fluorescence of isolated rat liver mitochondria," Biochim. Biophys. Acta $\mathbf{8 4 1}$, 237-246 (1985).

19. W. S. Kunz, "Evaluation of electron-transfer flavoprotein and alpha-lipoamide dehydrogenase redox states by two-channel fluorimetry and its application to the investigation of beta-oxidation," Biochim. Biophys. Acta 932, 8-16 (1988).

20. W. S. Kunz, "Spectral properties of fluorescent flavoproteins of isolated rat liver mitochondria," FEBS Lett. 195, 92-96 (1986).

21. C. I. Ragan, P. B. Garland, "Spectroscopic studies of flavoproteins and non-haem iron proteins of submitochondrial particles of torulopsis utilis modified by iron- and sulphate-limited growth in continuous culture," Biochem. J. 124, 171-187 (1971).

22. K. H. Vuorinen, A. Ala-Rämi, Y. Yan, P. Ingman, I. E. Hassinen, "Respiratory control in heart muscle during fatty acid oxidation. Energy state or substrate-level regulation by $\mathrm{Ca}^{2+}$ ? " J. Mol. Cell. Cardiol. 27, 1581-1591 (1995).

23. A. Ala-Rämi, M. Ylihautala, P. Ingman, I. E. Hassinen, "Influence of calcium-induced workload transitions and fatty acid supply on myocardial substrate selection," Metabolism 54, 410-420 (2005).

24. J. Koziol, "Fluorometric analyses of riboflavin and its coenzymes," Methods Enzymol. 18, 253-285 (1971).

25. A. de Kok, A. J. Visser, "Flavin binding site differences between lipoamide dehydrogenase and glutathione reductase as revealed by static and timeresolved flavin fluorescence," FEBS Lett. 218, 135138 (1987).

26. A. K. Lam, P. N. Silva, S. M. Altamentova, J. V. Rocheleau, "Quantitative imaging of electron transfer flavoprotein autofluorescence reveals the dynamics of lipid partitioning in living pancreatic islets," Integr. Biol. (Camb.) 4, 838-846 (2012). 
27. T. Bücher, B. Brauser, A. Conze, F. Klein, O. Langguth, H. Sies, "State of oxidation-reduction and state of binding in the cytosolic NADH-system as disclosed by equilibration with extracellular lactate-pyruvate in hemoglobin-free perfused rat liver," Eur. J. Biochem. 27, 301-317 (1972).

28. J. R. Sparrow, E. Gregory-Roberts, K. Yamamoto, A. Blonska, S. K. Ghosh, K. Ueda, J. Zhou, "The bisretinoids of retinal pigment epithelium," Prog. Retin. Eye Res. 31, 121-135 (2012).

29. K. Arai, T. Kanaseki, S. Ohkuma, "Isolation of highly purified lysosomes from rat liver: Identification of electron carrier components on lysosomal membranes," J. Biochem. 110, 541-547 (1991).

30. H. J. Shin, J. L. Mego, "A rat liver lysosomal membrane flavin-adenine dinucleotide phosphohydrolase: Purification and characterization," Arch. Biochem. Biophys. 267, 95-103 (1988).

31. B. Chance, B. Schoener, R. Oshino, F. Itshak, Y. Nakase, "Oxidation-reduction ratio studies of mitochondria in freeze-trapped samples. NADH and flavoprotein fluorescence signals," J. Biol. Chem. 254, 4764-4771 (1979).

32. B. Quistorff, B. Chance, "Simple techniques for freeze clamping and for cutting and milling of frozen tissue at low temperature for the purpose of two- or three-dimensional metabolic studies in vivo," Anal. Biochem. 108, 237-248 (1980).

33. B. Quistorff, H. Poulsen, "Evaluation of a freezeclamping technique designed for two- and threedimensional metabolic studies of rat liver in vivo. quenching efficiency and effect of clamping on tissue morphology," Anal. Biochem. 108, 249-256 (1980).

34. L. Z. Li, R. Zhou, T. Zhong, L. Moon, E. J. Kim, H. Qiao, S. Pickup, M. J. Hendrix, D. Leeper, B. Chance, J. D. Glickson, "Predicting melanoma metastatic potential by optical and magnetic resonance imaging," Adv. Exp. Med. Biol. 599, 67-78 (2007).

35. H. N. Xu, G. Zheng, J. Tchou, S. Nioka, L. Z. Li, "Characterizing the metabolic heterogeneity in human breast cancer xenografts by 3D high resolution fluorescence imaging," Springerplus 2, 73 (2013).

36. M. Mokry, P. Gal, B. Vidinsky, J. Kusnir, K. Dubayova, S. Mozes, J. Sabo, "In vivo monitoring the changes of interstitial $\mathrm{pH}$ and $\mathrm{FAD} / \mathrm{NADH}$ ratio by fluorescence spectroscopy in healing skin wounds," Photochem. Photobiol. 82, 793-797 (2006).

37. M. C. Skala, A. Fontanella, L. Lan, J. A. Izatt, M. W. Dewhirst, "Longitudinal optical imaging of tumor metabolism and hemodynamics," J. Biomed. Opt. 15, 011112 (2010).

38. M. Minsky, "Memoir on inventing the confocal scanning microscope," Scanning 10, 128-138 (1988).

39. K. M. Berland, P. T. So, E. Gratton, "Two-photon fluorescence correlation spectroscopy: Method and application to the intracellular environment," Biophys. J. 68, 694-701 (1995).

40. A. V. Kuznetsov, J. Troppmair, R. Sucher, M. Hermann, V. Saks, R. Margreiter, "Mitochondrial subpopulations and heterogeneity revealed by confocal imaging: Possible physiological role?" Biochim. Biophys. Acta 1757, 686-691 (2006).

41. F. Appaix, A. V. Kuznetsov, Y. Usson, L. Kay, T. Andrienko, J. Olivares, T. Kaambre, P. Sikk, R. Margreiter, V. Saks, "Possible role of cytoskeleton in intracellular arrangement and regulation of mitochondria," Exp. Physiol. 88, 175-190 (2003).

42. A. V. Kuznetsov, O. Mayboroda, D. Kunz, K. Winkler, W. Schubert, W. S. Kunz, "Functional imaging of mitochondria in saponin-permeabilized mice muscle fibers," J. Cell. Biol. 140, 1091-1099 (1998).

43. W. S. Kunz, K. Winkler, A. V. Kuznetsov, H. Lins, E. Kirches, C. W. Wallesch, "Detection of mitochondrial defects by laser fluorimetry," Mol. Cell. Biochem. 174, 97-100 (1997). 